\title{
A comparison of duloxetine hydrochloride with fluoxetine hydrochloride in major depressive disorders: a pilot study
}

\author{
Ravinder Kumar Sah ${ }^{1}$, Harmeet Singh Rehan ${ }^{2}$, Kannanore Eloremadathil Sadanandan Unni ${ }^{3}$, \\ Deepti Chopra ${ }^{1 *}$, Seema Manak ${ }^{1}$, Preeta Kaur Narula ${ }^{1}$

\footnotetext{
${ }^{1}$ Senior resident, Department of Pharmacology, Lady Hardinge Medical College, New Delhi, India;

*Corresponding Author: drdeeptichopra@yahoo.co.in

${ }^{2}$ Professor and Head, Department of Pharmacology, Lady Hardinge Medical College, New Delhi, India

${ }^{3}$ Professor and Head, Department of Psychiatry, Lady Hardinge Medical College, New Delhi, India
}

Received 23 December 2009; revised 11 January 2010; accepted 13 January 2010.

\begin{abstract}
To compare remission rate, relapse rate and tolerability of duloxetine, a dual reuptake inhibitor of 5-hydroxy serotonin (5-HT) and norepinephrine (NE), versus fluoxetine, a reuptake inhibitor of 5-HT during follow up period of 16 weeks in major depressive disorder (MDD) a open label comparative trial was conducted. Trial was comprising of $\mathbf{6 0}$ patients, diagnosed with MDD, were allocated to fluoxetine group $\left(n^{*}-30,20-60 m g\right.$ od) or duloxetine group ( $n^{*}-30$, $40-60 \mathrm{mg}$ od) for 16 weeks. The end points were remission and relapse assessed by Hamilton Rating scale for Depression-24 items (HAMD-24). In results the mean fall in HAMD-24 scores between groups was comparable till 4 weeks. Thereafter, at 8 weeks the mean fall in HAMD-24 score was significantly greater in duloxetine group ( $p$ value $<0.05$ ). At 16 weeks the mean fall was highly significant ( $p$ value $<0.01$ ) in duloxetine group. Thirty percent patients in duloxetine group achieved remission in comparison to none in fluoxetine group. None of the patient, in any group, reported relapse. Adverse effects were mild to moderate in severity.In conclusion duloxetine has a better pharmacological profile over fluoxetine in terms of efficacy and safety.
\end{abstract}

Keywords: Major Depressive Disorder; Duloxetine; Remission; Relapse

\section{INTRODUCTION}

Major depressive disorder (MDD) represents one of the most serious conditions encountered in clinical practice affecting 340 million people globally [1]. It is currently estimated to be the fourth leading cause of disability adjusted life years (DALY) in all age groups. Selective serotonin reuptake inhibitors (SSRIs) are the treatment of choice for moderate to severe depression [2]. However, studies have shown that up to half of all depressed patients fail to respond to SSRIs treatment. Thus there exists need for alternatives with multiple mechanisms along with avoidance of unwanted effects of tricyclic antidepressants (TCA) [3].

Duloxetine, a newer antidepressant which belongs to selective serotonin nor-epinephrine reuptake inhibitor (SSNRI) group, has been approved for MDD [4]. In double blind, placebo controlled multicentre, randomized, 9 weeks trials Gartlehner et al. [5] and Detke et al. [6] concluded that, for treatment of MDD Duloxetine is safe and effective. In 8 weeks, fluoxetine comparator trial, remission rates were high in duloxetine group, but not statistically significantly than fluoxetine group [7]. For long term treatment of MDD duloxetine has been shown to be effective in 52 weeks non-comparator open label multicentre trial [8].

As previous studies indicate that duloxetine may have some advantages in MDD, however there are limited numbers of studies which assess SSRIs as active comparators [9]. These studies are lacking with data's either duo to of short duration of study or duo to of lack of standard comparator. Therefore the current study was planned to compare remission rate, relapse rate and safety of oral duloxetine (SSNRI) with fluoxetine (SSRI) in 16 weeks comparative study.

\section{MATERIALS AND METHODS}

An open label study was conducted in the Department of 
Pharmacology in collaboration with the Department of Psychiatry, Lady Hardinge Medical College and associated Smt. Sucheta Kriplani (SSK) Hospital, New Delhi. Sixty six Patients, aged between 18 and 65 years with either sex, presenting with MDD, as per ICD-10 DCR criteria [10], on an outpatient basis, were included after obtaining an informed consent. The study protocol was approved by Institution Ethics Committee. Baseline disease severity was defined by patients' scores on the Hamilton Rating Scale for Depression-24 items (HAMD24) [11]. Exclusion criteria were history of psychosis or bipolar disorder, substance abuse disorder, intake of psychoactive medication during past two weeks. Patients with serious medical illness, serious suicidal risk requiring hospitalization and pregnant or lactating women were also excluded from study. A complete workup was done for all patients with respect to history, baseline investigations (weight, blood pressure, hemoglobin, complete blood count, blood sugar, serum electrolyte, kidney and liver function test) and clinical examinations.

All patients were randomly assigned to Fluoxetine 20-60 mg per oral (FLX) and Duloxetine 40-60 mg per oral (DLX) group. Both the study drugs were administered daily for 16 weeks and dose was titrated as per the need of patient.

\section{CLINICAL ASSESSMENT OF PATIENTS}

Clinical response of the patients was assessed at baseline ( 0 week), 1 week, 2 week, 4 week, 8 week and 16 week by using HAMD-24 scale. Improvement in HAMD-24 score from baseline was used as the primary efficacy measure to assess remission and relapse. Remission was defined as a total score on HAMD-24 of $\leq 7$ and relapse was defined as an episode of MDD that occurs within 6 months after either response or remission [12].

\section{SAFETY PROFILE}

The patients were monitored for any adverse drug effects during the course of study. The patients as well as the informants were asked in detail about any adverse drug effects the course of treatment on each visit.

\section{STATISTICAL ANALYSIS}

Baseline and end-point HAMD-24 scores were compared within groups using the paired student's t-test. Unpaired student's t-test was used for comparison of parameters between two study groups. Safety profile and response rate were compared between two treatment groups by Fisher's exact test. $\mathrm{P}$ value of $<0.05$ was taken to be significant.

\section{RESULTS}

A total of 66 patients enrolled, out of which 60 completed the study. Biosocial and demographic characteristics of patients were comparable (Table 1). Baseline bodyweight, pulse rate, blood pressure and biochemical parameters in both the groups were matching. On completion of treatment there were reduction in mean body weight in both groups which was statistically significant $(\mathrm{P}<0.05)$. In FLX group bodyweight reduced from $59.27 \pm 1.93 \mathrm{~kg}$ to $58.84 \pm 1.84 \mathrm{~kg}(\mathrm{p}$ value $<0.05)$ while in DLX group body weight reduced from $57.37 \pm$ $1.53 \mathrm{~kg}$ to $56.77 \pm 1.52 \mathrm{~kg}$ after 16 weeks. In DLX group blood sugar levels also reduced significantly ( $\mathrm{p}$ value $<$ 0.05 ) from $104.60 \pm 2.33 \mathrm{mg} / \mathrm{dl}$ to $102.73 \pm 2.03 \mathrm{mg} / \mathrm{dl}$ while there was statistically significant rise in hemoglobin at end of the study. The mean values of other parameters after16 week were comparable within as well as between the both groups.

\section{CLINICAL RESPONSE (EFFICACY)}

Baseline HAMD-24 scores in FLX and DLX groups were $17.46 \pm 0.13$ and $17.70 \pm 0.08$ respectively (Table 2). In FLX group the HAMD-24 scores reduced signifycantly from $17.46 \pm 0.13$ to $16.16 \pm 0.19$ ( $\mathrm{p}$ value $<0.05$ ) after first week followed by $16.3 \pm 0.19,14.8 \pm 0.19,12$ \pm 0.15 and $9.9 \pm 0.14$ HAMD-24 scores respectively at 2 weeks, 4 week, 8 week and 16 week. Similarly in DLX group the score reduced significantly from $17.70 \pm 0.08$ to $16.80 \pm 0.21$ after first week, there after $15.96 \pm 0.16$, $14.86 \pm 0.20,11.10 \pm 0.24$ and $8.06 \pm 0.15$ HAMD-24

Table 1. Comparison of biosocial characteristics of study patients.

\begin{tabular}{lcc}
\hline \multicolumn{1}{c}{ Characteristics } & FLX & DLX \\
\hline No. of patients & 30 & 30 \\
Age (years) Mean \pm SEM & $34.06 \pm 1.76$ & $32.50 \pm 2.01$ \\
Sex [n] & & \\
Male (n=18) & $12(40.0 \%)$ & $6(20.0 \%)$ \\
Female (n=42) & $18(60.0 \%)$ & $24(80.0 \%)$ \\
Marital status [n (\%)] & & \\
Married & $20(66.66 \%)$ & $20(66.66 \%)$ \\
Unmarried & $5(16.6 \%)$ & $8(26.6 \%)$ \\
Divorce & $5(16.6 \%)$ & $2(06.6 \%)$ \\
\hline
\end{tabular}


Table 2. Mean fall in HAMD-24 scores with time in both groups (Mean $\pm \mathrm{SEM}$ ).

\begin{tabular}{lcc}
\hline \multicolumn{1}{c}{ Time } & FLX & DLX \\
\hline Baseline (0 week) & $17.46 \pm 0.13$ & $17.70 \pm 0.08$ \\
1 week & $16.16 \pm 0.19$ & $16.80 \pm 0.21$ \\
2week & $16.30 \pm 0.19$ & $15.96 \pm 0.16$ \\
4 week & $14.83 \pm 0.19$ & $14.86 \pm 0.20$ \\
8 week & $12.06 \pm 0.15$ & $11.10 \pm 0.24^{*}$ \\
16 week & $9.96 \pm 0.14$ & $8.06 \pm 0.15^{* * *}$ \\
\hline
\end{tabular}

* $\mathrm{P}<0.05$ (Student's t-test), *** $\mathrm{P}<0.01$ (Student's t-test).

scores were recorded at 2 week, 4 week, 8 week and 16 week respectively.

On comparing FLX and DLX groups the mean fall in HAMD-24 scores were comparable till 4 weeks. Thereafter, at 8 weeks the mean fall in HAMD score was significantly greater in DLX group i.e. $11.10 \pm 0.24(\mathrm{p}<$ $0.05)$. At 16 weeks the mean fall was highly significant ( $p$ value $<0.01$ ) in DLX group vs. the FLX group (Table 2).

\section{REMISSION AND RELAPSE}

In FLX group there was no remission till 16 weeks of treatment, where as in DLX group 9 patients remitted with in 16 weeks. None of the patient, in any group, reported relapse of any sign or symptoms of MDD during study period.

\section{ADVERSE DRUG EVENTS}

A total of 99 adverse events were recorded in FLX group as compared to 37 adverse events in DLX group. Nausea was frequent adverse events in FLX group as compared to DLX. Insomnia, sweating and diarrhoea were the other frequent adverse events observed in FLX group. Nausea, dry mouth, diarrhoea and fatigue constituted the frequent adverse events in DLX group. These adverse effects were mild to moderate and there were no serious adverse event in both the groups (Table 3).

\section{DISCUSSION}

After one week of treatment, overall fall in HAMD-24 score was greater in DLX group than FLX group. In DLX (20 mg twice daily) group, reduction in HAMD-24 score at 8 and 16 weeks from baseline value was -6.6 and -9.64 whereas in FLX (20 mg once daily) fall was -5.4 and -7.5 . Similarly, Goldstein et al. (2002) reported a higher fall in HAMD scores with duloxetine
Table 3. Adverse drug events observed in the two treatment groups.

\begin{tabular}{lcc}
\hline \multicolumn{1}{c}{ Adverse Event } & FLX (n-99) & DLX (n-37) \\
\hline Nausea & $17(17.1 \%)$ & $8(21.6 \%)$ \\
Dry mouth & $7(7 \%)$ & $5(13.5 \%)$ \\
Constipation & $5(5 \%)$ & $4(10.8 \%)$ \\
Diarrhoea & $13(13.1 \%)$ & $4(10.8 \%)$ \\
Vomiting & $7(7 \%)$ & $2(5.4 \%)$ \\
Anorexia & $12(12.1 \%)$ & $2(5.4 \%)$ \\
Fatigue & $1(1 \%)$ & $3(8.1 \%)$ \\
Dizziness & $2(2 \%)$ & $3(8.1 \%)$ \\
Somnolence & 0 & $2(5.4 \%)$ \\
Sweating & $14(14.1 \%)$ & $3(8.1 \%)$ \\
Insomnia & $15(15.1 \%)$ & $1(2.7 \%)$ \\
Anxiety & $3(3 \%)$ & 0 \\
Ejaculation delayed & $3(3 \%)$ & 0 \\
\hline
\end{tabular}

(n- Number of total adverse events)

$(-9.73)$ than with fluoxetine $(-7.75)$ or placebo $(-6.6)$ in an 8 week study [7]. Hudson et al. (2007) reported higher reduction in HAMD-24 scores at 12 weeks with duloxetine $(60 \mathrm{mg} /$ day $)$ [1]. The early and higher response rate may be due to higher dose of duloxetine. In present 16 week study and previous short period studies, duloxetine shown to have better efficacy than SSRIs, which may be due to its combined effects on both the serotonin and norepinephrine systems. This dual mechanism makes duloxetine potent (4.5 times) and to act faster than SSRIs $[1,13]$. Also, when compared with other dual action antidepressants like venlafaxine, duloxetine appears to be more balanced inhibitor of serotonin and norepinephrine reuptake. Further, experimental data predicted that dual serotonin and norepinephrine exert analgesic effects via descending pain pathways; therefore duloxetine is more suitable in depression with somatic symptoms [1].

In this study response rate from one week onward was $100 \%$ in both the groups and remission rate was 33\% and $0 \%$ in DLX and FLX group respectively at end of 16 weeks. Goldstein D.J. et al. (2002) also reported response and remission rate of $64 \%$ and $56 \%$ respectively for du- loxetine (20 mg twice daily), compared with $52 \%$ and $30 \%$ for fluoxetine ( $20 \mathrm{mg}$ once daily) and $48 \%$ and $32 \%$ for placebo, indicates superior efficacy of duloxetine over Fluoxetine [7]. In another 8 weeks trial, Goldstein DJ (2004) showed response and remission rates were $54 \%$ and $37 \%$ for duloxetine ( $40 \mathrm{mg}$ once daily), $60 \%$ and $58 \%$ for duloxetine $(80 \mathrm{mg}$ ) in comparison to 
$50 \%$ and $34 \%$ for paroxetine ( $20 \mathrm{mg}$ once daily) and $30 \%$ and $28 \%$ for placebo [14]. In a metanalysis by Jennifer et al. (2006) the remission rate was found to be superior for duloxetine as compared to SSRIs ( $43 \%$ vs. $38 \%$ ). Also when alternative definition of remission (MADRS score $\leq 12$ ) was used, duloxetine was found to be sig- nificantly better than SSRIs [9].

In both groups there was no relapse till 16 weeks. Although the study duration of 4 months is not sufficient to comment upon relapse rate but as per definition of relapse there was no reoccurrence of sign and symptoms after response has been establish in both groups. Relapse rate for the fluoxetine group was found to be $35.2 \%$ after 6 month and $45.9 \%$ after 1 year of treatment [15]. In randomized double blind, multicenter, placebo controlled, parallel group study, David G.P. et al. (2006) showed that duloxetine (60 mg once daily) was effective in the prevention of relapse of MDD during continuation of treatment [16]. Geddes et al. (2003) had concluded on systemic review of 31 antidepressant trials that continuing with antidepressant reduced the odds of depressive relapse by $70 \%$ compared to placebo. This suggests continued antidepressant treatment has ability to reduce the chances of depressive relapse [17].

Duloxetine has better adverse effect profile. In DLX group $30 \%$ of patients experienced adverse events in which nausea $(26.7 \%)$ and dry mouth $(16.7 \%)$ were principally reported. Other adverse events were diarrhea, fatigue, dizziness and vomiting. Sharma A. et al. (2000) have also reported similar adverse effect profile for duloxetine [18]. As a reuptake inhibitor of norepinephrine, duloxetine has been found to be associated with mean increase in heart rate as well as blood pressure (less than $2 \mathrm{mmHg}$ ) [8]. No such cardiovascular adverse effect was observed in this study .In FLX group, patients mainly reported nausea $(56.6 \%)$ and insomnia $(50 \%)$ followed by other adverse events which includes sweating, diarrhea, anorexia, vomiting, dry mouth and anxiety. In a study of 6 months, Zajecka et al. (1999) have reported similar adverse events [19].

Ejaculation delayed was reported by 3 male patients $(10 \%)$ in FLX group. Similarly Harman J.B. et al. (1990) also reported frequent sexual dysfunction with Fluoxetine [20]. Comparatively in DLX group, there was no sexual dysfunction occurred in any patients although it has been reported by Detke M.J. et al. (2002) [21].

All the adverse effects during study period were mild to moderate severity. On the WHO casualty assessment scale they were classified as possible. Favorable adverse effect profile of duloxetine may be because of its less affinity for dopaminergic, adrenergic, cholinergic, histaminergic, opioid, glutamate and GABA receptors [1].

\section{CONCLUSIONS}

In this comparative study duloxetine was found to be more efficacious than fluoxetine in reducing HAMD-24 score in 16 weeks as well as found to have better adverse effect profile than fluoxetine. This suggests that for treatment of MDD duloxetine is better option over fluoxetine.

\section{REFERENCES}

[1] Hudson, J.I., Perahia, D.G., Gilaberte, I., Wang, F., Watkin, J.G. and Detke, M.J. (2007) Duloxetine in the treatment of major depressive disorder: An open-label study. BMC Psychiatry, 7(1), 43. http://www.biomedcentral.com

[2] Williams, R.H.M. (2005) Duloxetine (Cymbalta): a new treatment option in depression. Prescriber, 16(12), 49-50. http://www.escriber.com

[3] McIntyre, R.S., Muller, A., Mancini, D.A. and Silver, E.S. (2003) What to do it an initial antidepressant fails. $\mathrm{Ca}$ nadian Family Physician, 49(4), 449-457.

[4] Gartlehner, G., Hansen, R.A., Kahwati, L., et al. (2006) Drug class review on second generation antidepressants Final Report March. Oregan Evidance based Practice Centre, Oregan Health \& Science University, Portland.

[5] Detke, M.J., Lu, Y., Goldstein, D.J., Hayes, J.R. and Demitrack, M.A. (2002) Duloxetine, $60 \mathrm{mg}$ once daily for major depressive disorder: a randomized double blind placebo controlled trial. Journal of Clinical Psychiatry, 63(4), 308-315.

[6] Detke, M.J., Lu, Y., Goldstein, D.J., McNamara, R.K. and Demitrack, M.A. (2002) Duloxetine, $60 \mathrm{mg}$ once daily dosing versus placebo in the acute treatment of major depression. Journal of Psychiatric Research, 36(6), 383-390.

[7] Goldstein, D.J., Mallinckrodt, C., Lu, Y., Demitrack and M.A. (2002) Duloxetine in the treatment of major depressive disorder: A double blind clinical trial. Journal of Clinical Psychiatry, 63(3), 225-231.

[8] Raskin, J., Goldstein, D.J., Mallinckrodt, C.H. and Ferguson, M.B. (2003) Duloxetine in the long term treatment of major depressive disorder. Journal of Clinical Psychiatry, 64(10), 1237-1244.

[9] Kirwin, J.L. and Gören, J.L. (2005) Duloxetine: a dual serotonin-norepinephrine reuptake inhibitor for treatment of major depressive disorder. Pharmacotherapy, 25(3), 396-410.

[10] Depressive Episode F-32 (1992) The ICD-10 classification of mental and behavioural disorders, diagnostic criteria for research. World Health Organization, Geneva. 81-85.

[11] Blacker, D. (2005) Psychiatric rating scales. In. Kaplan, H.I. and Sadock, B.J. Eds. Comprehensive textbook of Psychiatry, 8th Edition, Williams and Wilkins, Baltimore.

[12] Segal, Z.V., Pearson, J.L. and Thase, M.E. (2003) Challenges in preventing relapse in major depression: Report of a National Institute of Mental Health Workshop on state of the science of relapse prevention in major de- 
pression. Journal of Affective Disorders, 77(2), 97-108.

[13] Schweitzer, I., Burrows, G. and Tuckwell, V., et al. (2001) Sustained response to open-label venlafaxine in drug-resistant major depression. Journal of Clinical Psychopharmacology, 21(2), 185-189.

[14] Goldstein, D.J., Lu, Y., Detke, M.J., Wilste, C., Mallinckrodt, C. and Demitrack, M.A. (2004) Duloxetine in the treatment of depression: A double blind placebo controlled comparison with paroxetine. Journal of Clinical Psychopharmacology, 24(4), 389-399.

[15] McGrath, P.J., Stewart, J.W., Quitkin, F.M., et al. (2006) Predictors of Relapse in a Prospective Study of Fluoxetine Treatment of Major Depression. American Journal of Psychiatry, 163 (9), 1542-1548.

[16] Perahia, D.G., Gilaberte, I., Wang, F., et al. (2006) Duloxetine in the prevention of relapse of major depressive disorder: a double blind placebo controlled study. British Journal of Psychiatry, 188, 346-353.

[17] Geddes, J.R., Carney, S.M., Davis, C., et al. (2003) Relapse prevention with antidepressant drug treatment in depressive disorders: A systemic review. Lancet, 361 (9358), 653-661.

[18] Sharma, A., Goldberg, M.J. and Cerimele, B.J. (2000) Pharmacokinetics and safety of duloxetine, a dual serotonin and norepinephrine reuptake inhibitor. Journal Clinical Pharmacology, 40(2), 161-167.

[19] Zajecka, J., Amsterdam, J.D., Quitkin, F.M., et al. (1999) Changes in adverse events reported by patients in six months of fluoxetine therapy. Journal of Clinical Psychiatry, 60(6), 389-394.

[20] Harman, J.B., Brotman, A.W., Pollock, M.H., Falk, W.E., Biederman, J. and Rosenbaum, J.F. (1990) Flouxetine induced sexual dysfunction. Journal of Clinical Psychiatry, 51(1), 25-27.

[21] Detke, M.J., Lu, Y., Goldstein, D.J., Hayes, J.R. and Demitrack, M.A. (2002) Duloxetine, $60 \mathrm{mg}$ once daily for major depressive disorder: A randomized double blind placebo controlled trial. Journal of Clinical Psychiatry, 63(4), 308-315. 